\title{
STATISTICAL SIMULATION OF NON-CIRCULAR CROSS SECTION POISEUILLE FLOWS
}

\author{
Jian-Zheng Jiang, Ching Shen, and Jing Fan \\ Laboratory of High Temperature Gas Dynamics, Institute of Mechanics, CAS, Beijing 100080, China
}

\begin{abstract}
This paper investigates the Poiseuille flows for rectangular, regular hexagonal, and semicircular cross sections in transition regime using particle approaches, namely the direct simulation Monte Carlo (DSMC) method and the information preservation (IP) method. The DSMC and IP results compare well with each other, while the IP method is much more computationally efficient than the DSMC method. The mass flow rates given by IP and DSMC are in agreement with the BGK solutions of Hasegawa and Sone. For rectangular cross sections in the wide range of the width-to-height ratio, the simulation results of the mass flow rates and the velocity profiles along the midperpendicular line have been given for both methods to estimate the lateral wall influence. For the physical quantities, such as the mass flow rate, which are influenced by the whole field, the lateral wall influence must be considered even for width-to-height ratio as large as 10. And for the physical parameters, such as the maximum velocity and the velocity profile along the midperpendicular line, the lateral wall influence can be negligible if the width-to-height ratio is bigger than 5 .
\end{abstract}

\section{INTRODUCTION}

The rarefied gas flows through a pipe can be used in many technologies such as vacuum equipment, micro-electro-mechanical systems (MEMS), spaceship construction etc. The Poiseuille flow problem considers the flow in a long pipe under the action of pressure difference imposed on the two ends of the pipe in the assumption of linear pressure distribution, and has provoked interest of many scientists and engineers (Cercignani and Sernagiotto, 1966; Sone and Yamamoto, 1968; Loyalka et al., 1976; Hasegawa and Sone, 1988; Ohwada et al., 1989; Sharipov, 1999; Aoki, 2001). Most of the work is devoted to the flows between two parallel plates and the flows through circular capillaries. But some studies are carried on non-circular cross sections, such as rectangle, regular hexagon, semicircle etc. Recently, a new fabrication technique, micromachining, is used to make microchannels. And the rectangular cross section is of special importance, as the bulk-etching technique produces microchannel with such cross section. Recent experiment investigations also have revealed the non-linearity of the pressure distribution (Pong et al., 1994; Shih et al., 1996). But as the consideration under linear pressure assumption can capture the main features of the flow, it still deserves investigation as a fundamental problem.

When the size of the systems is so small, or the gas is so dilute, that the mean free path of the gas molecules is not negligibly small compared with the characteristic length of the systems, the ordinary fluid dynamics is not applicable, and a molecular gas dynamics approach based on kinetic theory is necessary. The results for the flow through a pipe with non-circular cross section available now are those obtained by the BGK model of the Boltzmann equation (see Loyalka et al., 1976; Hasegawa and Sone, 1988; Sharipov, 1999; and also the review paper Aoki, 2001). However the BGK model cannot get the true relationship of the transport properties corresponding to pressure and temperature in physical process. And some expedient measures must be taken to convert the results so that they can compare with the physical reality.

In the present paper, the direct simulation Monte Carlo (DSMC) method (Bird, 1994) and the information preservation (IP) method (Fan and Shen, 1999; Fan and Shen, 2001) are used to simulate the Poiseuille flows for non-circular cross sections in the transitional regime. The mass flow rates are compared with the results by the BGK model (Hasegawa and Sone, 1988); The flow fields obtained by the DSMC and the IP methods are compared with each other; For rectangular cross sections in the wide range of the width-to-height ratio, the mass flow rates and the velocity profiles along the midperpendicular line have been also given. In the next section, the DSMC and IP 
methods are briefly introduced; then the computational results are given followed by some discussions.

\section{THE DSMC METHOD AND THE IP METHOD}

The direct simulation Monte Carlo (DSMC) method (Bird, 1994) developed by G. A. Bird has been widely used to simulate the flows of low-density regimes. It is a molecular-based numerical scheme and is physically appropriate for this kind of gas flows where non-continuum, rarefied gas effects become important. The method has been validated by theory and experiments.

The essential approximation of DSMC method is the uncoupling, over a small time interval, of the molecular motion and the intermolecular collisions. In the DSMC procedures, macroscopic observable quantities, such as velocity and temperature, are obtained through averaging appropriate magnitudes of the simulated molecules over a small space region.

Because the statistical fluctuation in the DSMC method becomes insurmountably large at the low Mach numbers that are characteristic of MEMS, where the low macroscopic speed causes a large noise to useful information ratio, and a very large sampling size is demanded, the information preservation (IP) method is proposed to reduce the statistical fluctuation (Fan and Shen, 1999; Fan and Shen, 2001). In the method (Fan and Shen, 1999), simulated molecules move through physical space and undergo collisions appropriate to the thermal velocities using the same algorithms and models as the DSMC method (Bird, 1994), while the macroscopic observable quantities, such as velocity and surface shear stress, are obtained through averaging appropriate information quantities carried along with the simulated molecules. The information quantities reflect the collective behavior of the enormous number of real molecules represented by each simulated molecule in the DSMC method, and therefore are not subject to the statistical noise caused by the thermal velocity.

The IP method was successfully applied to many problems (Cai et al., 2000; Fan et al., 2001; Sun et al., 2001; Sun and Boyd, 2002; Xie et al., 2002; Shen et al., 2003) and was elucidated in detail in the newly published book (Shen, 2003). The results given by the method compare quite well with exact solutions of Navier-Stokes equations with slip boundary condition and the theoretical solutions of free molecular flows at the continuum and free molecular limits, and with the numerical solutions of the linearized Boltzmann equation, experimental data and DSMC calculations in the transition regime.

\section{PROBLEMS AND RESUTS}

The rarefied gas flows in channels of various cross sections due to the pressure gradient have been simulated by the IP method and the DSMC method. The hard sphere model is employed to describe the interaction between molecules. And the Knudsen number $(\mathrm{Kn})$ of the flow considered ranges from 0.05 to 10.0 , where $\mathrm{Kn}$ is defined as $\lambda / \mathrm{L}, \lambda$ is the mean free path (Bird, 1994):

$$
\lambda=(16 / 5)\left(\mathrm{m} /\left(2 \pi \mathrm{kT}_{0}\right)\right)^{1 / 2}\left(\mu / \rho_{0}\right),
$$

where $\mathrm{m}$ is molecular mass of the gas, $\mathrm{k}$ is Boltzmann constant, $\mathrm{T}_{0}$ is the gas reference temperature, $\mu$ is the coefficient of viscosity, $\rho_{0}$ is the reference density and $\mathrm{L}$ is a representative dimension of the cross section (shown in Fig. 1). The pressure variation along the axis of the channel is supposed to be linear

$$
\mathrm{p}=\mathrm{p}_{0}\left(1+\alpha \mathrm{X}_{3} / \mathrm{L}\right),
$$

where $\mathrm{X}_{3}$ is the Cartesian coordinate along the axis of the channel and the origin of it is at the center of the cross section considered, the pressure of which is taken as the reference value $p_{0}=\rho_{0} R_{0}$, where $\mathrm{R}$ is the gas constant, and $\alpha=\left(\mathrm{L} / \mathrm{p}_{0}\right)\left(\mathrm{dp} / \mathrm{dX}_{3}\right)$ is a (negative) constant. And only gas flow in cells between two adjacent cross sections are considered. The acceleration that results from the pressure distribution is $a=-\alpha p_{0} /\left(\rho_{0} L\right)$, during each time step $\Delta t$, the velocity increment along the axis of the pipe is calculated as

$$
\Delta \mathrm{u}=-\left(\alpha \mathrm{p}_{0} /\left(\rho_{0} \mathrm{~L}\right)\right) \Delta \mathrm{t}
$$

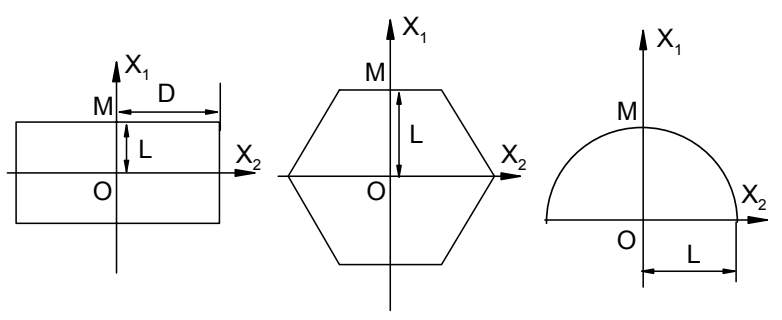

Fig. 1 Pipes with various cross sections

\section{The Mass Flow Rates For Various Cross Sections}

In Table 1 the IP and DSMC mass flow rates $\dot{M}$ for Rectangular, regular hexagonal, and semicircular cross sections at various $\mathrm{Kn}$ are given and compared with the results by the BGK model (Hasegawa and Sone, 1988). The mass flow rates are normalized by $-\alpha \mathrm{p}_{0} \mathrm{~S} /(2 \mathrm{RT})^{1 / 2}$, where $\mathrm{S}$ is the area of the cross 
section of the pipe. The width-to-height ratio $(\mathrm{D} / \mathrm{L})$ of rectangular cross sections is 2 . The Knudsen number for the BGK model here is divided by the coefficient $1.27=3.992 / \pi$, for the collision frequency in BGK model must be modified to result in a correct viscosity expression (see Vincenti et al., 1965; Shen et al., 1994).

As shown in Table 1, the results of the three methods are in good agreement with each other at large Knudsen numbers $(\mathrm{Kn}=0.7874$ and $\mathrm{Kn}=7.8740)$. The results of the IP and DSMC methods at smaller Knudsen number $(\mathrm{Kn}=0.07874)$ are smaller than the data obtained by the BGK model for rectangular cross section. This trend agrees with the fact that the flow rate obtained by the numerical solution of linearized Boltzmann equation for hard-sphere molecules (Ohwada et al., 1989) is smaller than the BGK results (Hasegawa and Sone, 1988) in the analysis of the flows between two parallel plates. However the results of the IP and DSMC methods at the Knudsen number $(\mathrm{Kn}=0.07874)$ for regular hexagonal and semicircular cross sections are a little bigger then the results obtained by the BGK model.

TABLE 1 Mass flow rates $\dot{M}$ versus $\mathrm{Kn}$ for various cross sections.

\begin{tabular}{|c|c|c|c|c|}
\hline \multicolumn{2}{|r|}{$\mathrm{Kn}$} & 0.0787 & 0.7874 & 7.8740 \\
\hline \multirow{3}{*}{$\begin{array}{c}\text { rectangle } \\
(\mathrm{D}=2 \mathrm{~L})\end{array}$} & IP & 6.505 & 2.230 & 2.073 \\
\hline & DSMC & 6.647 & 2.259 & 2.076 \\
\hline & $\begin{array}{l}\text { BGK(Hasegawa } \\
\text { and Sone, 1988) }\end{array}$ & 6.718 & 2.278 & 2.088 \\
\hline \multirow{3}{*}{$\begin{array}{l}\text { regular } \\
\text { hexagon }\end{array}$} & IP & 3.909 & 1.529 & 1.455 \\
\hline & DSMC & 3.972 & 1.546 & 1.455 \\
\hline & $\begin{array}{l}\text { BGK(Hasegawa } \\
\text { and Sone, 1988) }\end{array}$ & 3.885 & 1.550 & 1.461 \\
\hline \multirow{3}{*}{ semicircle } & IP & 1.7326 & 0.9211 & 0.9510 \\
\hline & DSMC & 1.7481 & 0.9231 & 0.9499 \\
\hline & $\begin{array}{l}\text { BGK(Hasegawa } \\
\text { and Sone, 1988) }\end{array}$ & 1.6874 & 0.9289 & 0.9545 \\
\hline
\end{tabular}

\section{The Flow Field For Various Cross Sections}

The IP and DSMC simulated isovels for rectangular cross section $(\mathrm{D}=2 \mathrm{~L})$ for $\mathrm{Kn}=0.0787$ are presented in Fig. 2 . The velocities are normalized by $-\alpha(2 \mathrm{RT})^{1 / 2}$. The IP and DSMC simulated isovels for regular hexagonal cross section for $\mathrm{Kn}=0.7874$ and for semicircular cross section for $\mathrm{Kn}=7.8740$ are shown in Fig. 3 and Fig. 4, respectively. The velocities are also normalized. From these figure, the good agreement of the results of the IP and DSMC methods can be found.

The normalized IP and DSMC simulated velocity profiles along the midperpendiculars $\mathrm{OM}$ for various cross sections are presented in Fig. 5.

In the present calculations a rather large $\alpha=-0.02373$ is selected which corresponds to a Mach number of about 0.15 . Under such condition the DSMC process converges relatively rapidly, and the sample size of DSMC result is only one order of magnitude bigger than the IP sample size. For example, the sample size of the IP method is about $6 \times 10^{5}$ and that of the DSMC method is about $9 \times 10^{6}$ to obtain the results shown in Fig. 3. If a small flow velocity typical for micro-channel is selected (say, about $0.2 \mathrm{~m} / \mathrm{s}$ as in Ho et al., 1998), the Mach number and $\alpha$ will be very small, and DSMC would require a sample size $10^{4}$ times larger than IP (Fan and Shen, 2001).

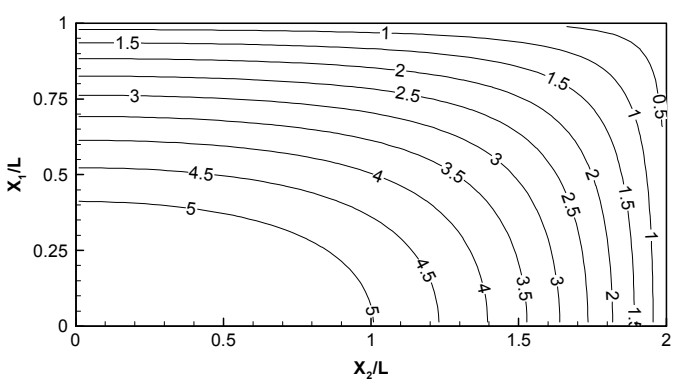

a) IP

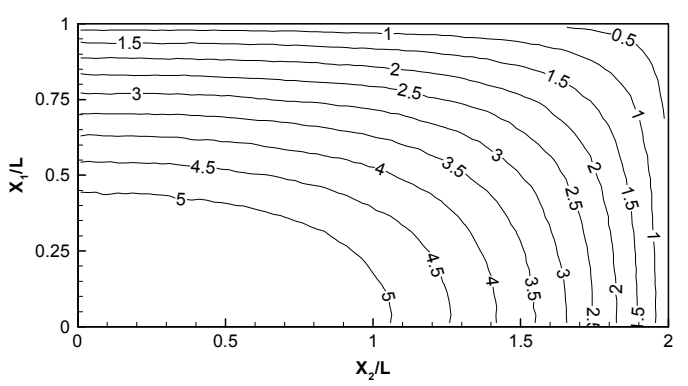

b) DSMC

Fig. 2 Isovels for rectangular cross section $(\mathrm{Kn}=0.0787)$ 


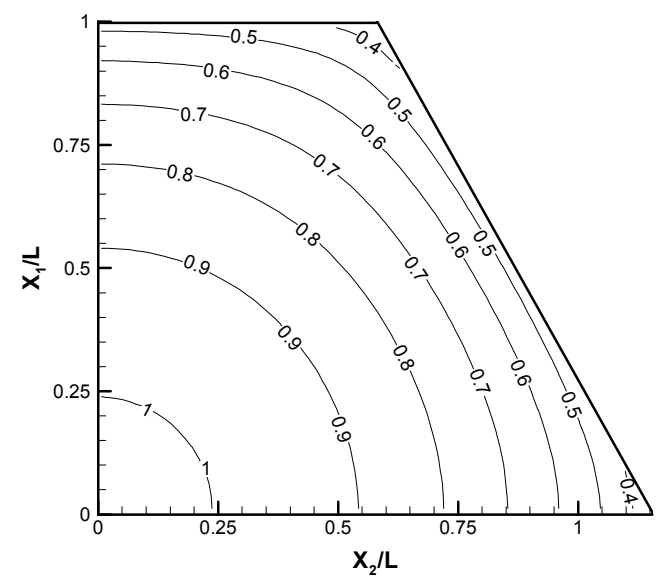

a) IP

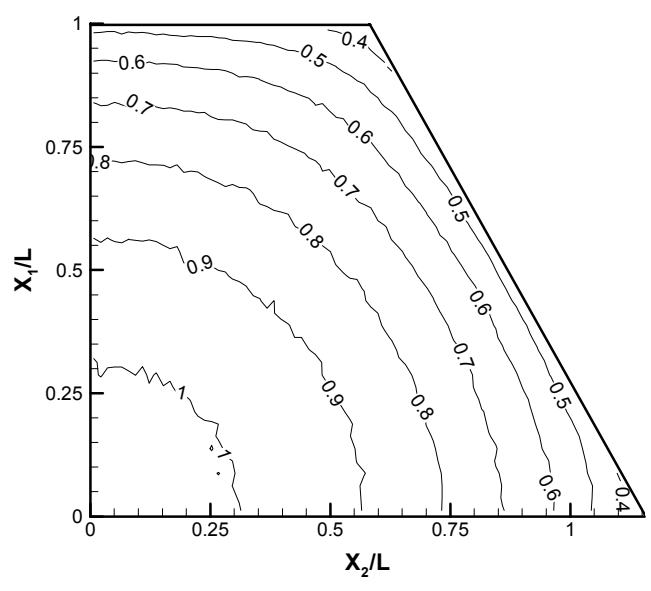

b) DSMC

Fig. 3 Isovels for regular hexagonal cross section $\quad(\mathrm{Kn}=0.7874)$

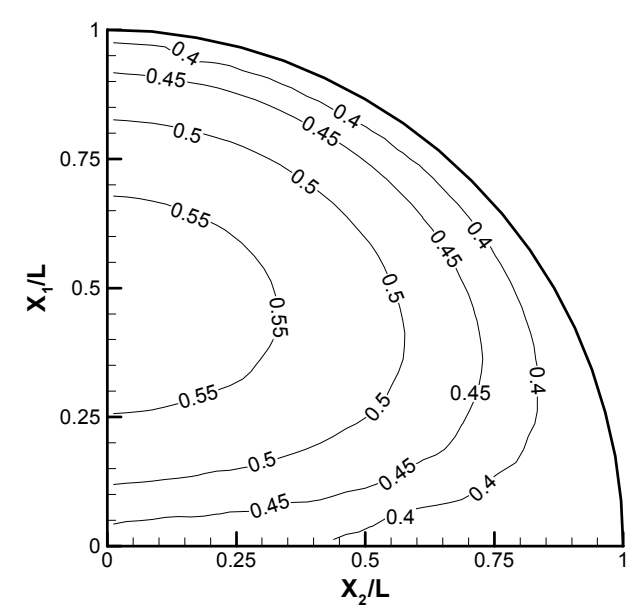

a) IP

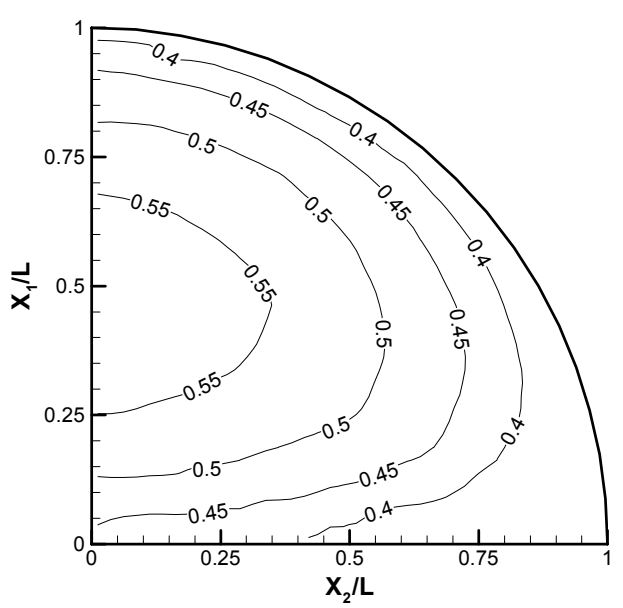

b) DSMC

Fig. 4 Isovels for semicircular cross section $(\mathrm{Kn}=7.8740)$

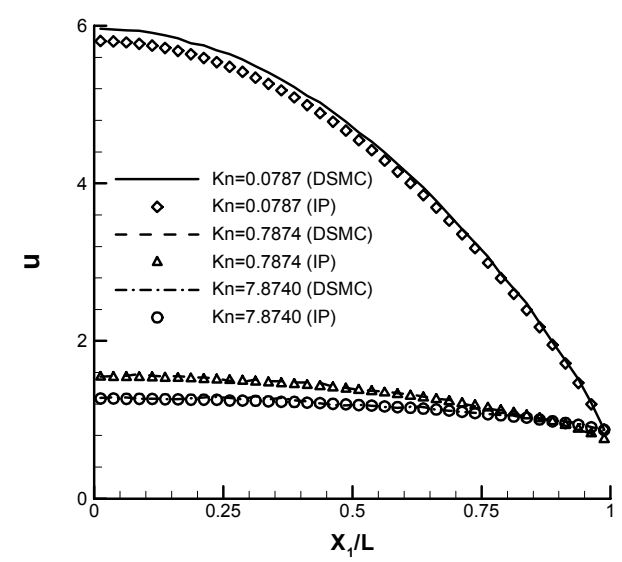

a) Rectangular cross section

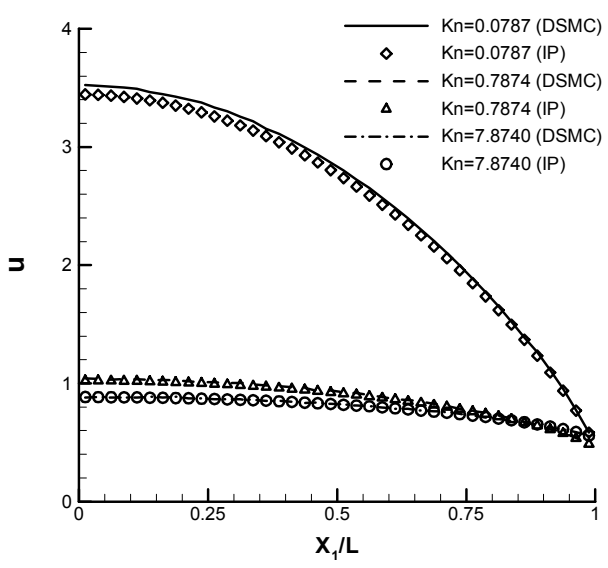

b) Regular hexagonal cross section 


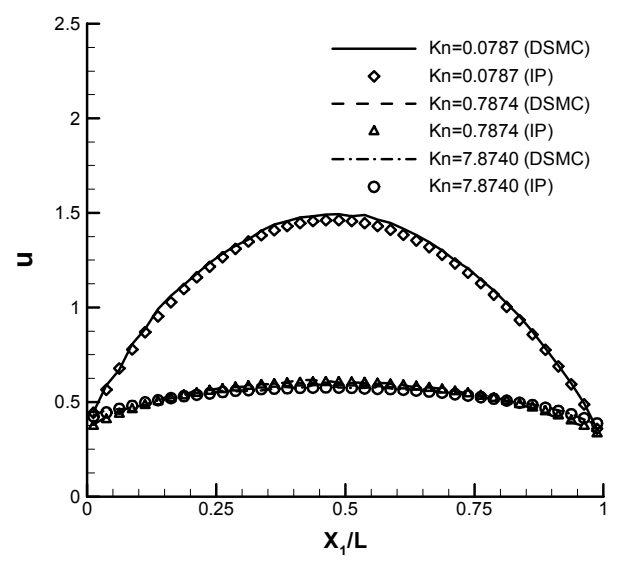

c) Semicircular cross section

Fig. 5 The velocity profiles along the midperpendiculars OM

\section{The Flows Through A Channel With Rectangular Cross Section And The Flows Between Two Infinite Plates}

In Table 2 the IP and DSMC mass flow rates $\dot{M}$ for various width-to-height ratios of rectangular cross section at $\mathrm{Kn}=0.2256$ are given. The mass flow rates are normalized by $-\alpha \mathrm{p}_{0} \mathrm{~S} /(2 \mathrm{RT})^{1 / 2}$. The normalized velocity profiles along the midperpendicular OM of the both methods for various width-to-height ratios are shown in Fig. 6.

TABLE 2 Mass flow rates $\dot{M}$ versus width-to-height ratios for rectangular cross section $(\mathrm{Kn}=0.2256)$

\begin{tabular}{|c|c|c|c|c|c|}
\hline $\begin{array}{l}\text { width-to-height } \\
\text { ratios }\end{array}$ & 1 & 2 & 5 & 10 & $\infty$ \\
\hline IP & 1.141 & 1.675 & 2.082 & 2.221 & 2.360 \\
\hline DSMC & 1.161 & 1.706 & 2.123 & 2.263 & 2.406 \\
\hline
\end{tabular}

From Table 2 one can see that the flow rates of finite width-to-height ratios are quite different from flow rate of an infinite width-to-height ratio (flow between two parallel plates). The discrepancy between the flow rates of a width-to-height ratio 10 and an infinite width-to-height ratio is about $7 \%$, which is in agreement with Sharipov's result (Sharipov, 1999). But Fig. 6 a) and b) show that the midperpendicular velocity profile and the maximum velocity of a rectangular cross section can be simulated quite well by the flow between two plates without side walls (width-to-height ratio $=\infty$ ), as long as the width-to-height ratio is bigger than 5. As the mass flow rate is a quantity obtained by integration over the whole field, so it is influenced by the slow down of the flow near the side walls, while the maximum velocity and the velocity profile along the midperpendicular line are local characteristics, and 5-10 width-to-height ratio is sufficient to ensure the minor influence of the side walls on the local quantities near the midperpendicular line.

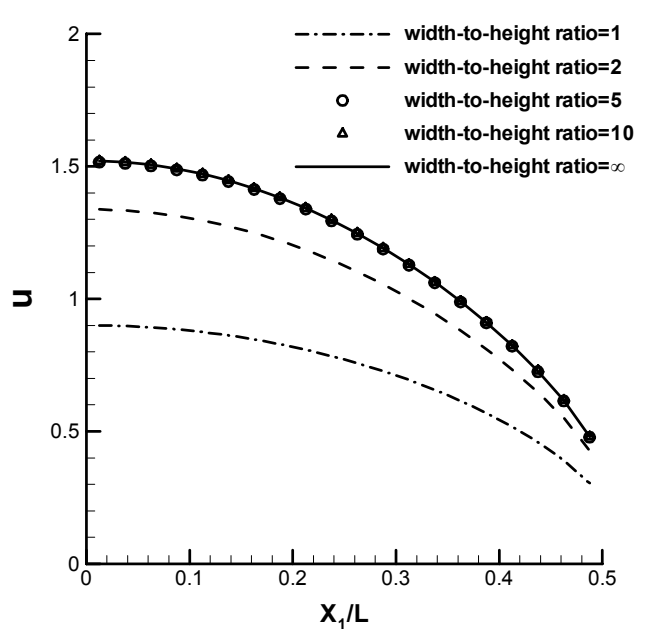

a) IP

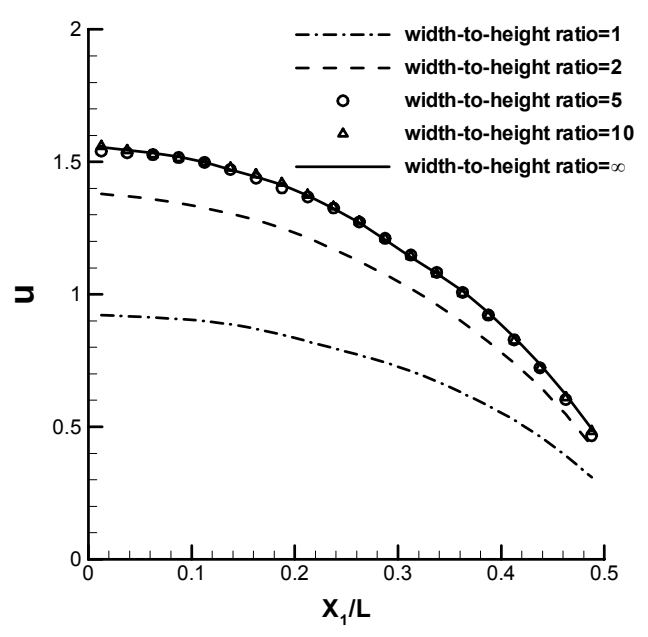

b) DSMC

Fig. 6 The velocity profiles along the midperpendicular $\mathrm{OM}$ of rectangular cross section for various width-to-height ratios $(\mathrm{Kn}=0.2256)$

\section{DISCUSSION}

The statistical simulation of non-circular cross section Poiseuille flows shows that the IP method can be applied to the flows in pipes with various cross sections as well as the DSMC method. The results, such as the mass flow rate and the velocity profile, are in agreement with data of the DSMC method. Meanwhile, the IP method reduces the sample size required by the DSMC 
method immensely for low speed flows. It can save computational time and lead to effective use of IP method for MEMS simulation.

The flow rate results of the IP method, the DSMC method and the BGK model method are in good agreement with each other at large Knudsen numbers $(\mathrm{K} n=0.7874$ and $\mathrm{Kn}=7.8740)$. The flow rate results of the IP and DSMC methods at smaller Knudsen number $(\mathrm{K} n=0.07874)$ are smaller than the data obtained by the BGK model for rectangular cross section. This trend agrees with the fact that the numerical solution of linearized Boltzmann equation for hard-sphere molecules (Ohwada et al., 1989) is smaller than the BGK results (Hasegawa and Sone, 1988) in the analysis of the flows between two parallel plates.

The statistical simulation for various width-to-height ratios of rectangular cross section shows that the threedimensional flows cannot be simulated as twodimensional flows to obtain the physical quantities, such as the mass flow rate, even by using the width-to-height ratio as large as 10 . And the simulation also shows that the two-dimensional flows can be used instead of three-dimensional flows to obtain the physical parameters, such as the maximum velocity and the velocity profile along the midperpendicular line, if the width-to-height ratio is bigger than 5 .

\section{ACKNOWLEDGMENT}

The authors appreciate support from NNSFC under Grants 19889209 and 90205024.

\section{NOMENCLATURE}

a the acceleration along the axis of the pipe, $\mathrm{m} / \mathrm{s}^{2}$

$\mathrm{k} \quad$ Boltzmann constant, $\mathrm{k}=1.3806 \mathrm{e}-23 \mathrm{~J} / \mathrm{K}$

$\mathrm{Kn} \quad$ Knudsen number, $\mathrm{Kn}=\lambda / \mathrm{L}$

$\lambda$ mean free path, $\mathrm{m}$

$\mathrm{L}$ a representative dimension of the cross section, $\mathrm{m}$

m molecular mass, $\mathrm{kg}$

$\dot{\mathrm{M}}$ the dimensionless mass flow rate

$\mathrm{P}_{0} \quad$ the reference pressure, $\mathrm{Pa}$

$\mathrm{R}$ the gas constant

$\mathrm{S}$ the area of the cross section of the pipe, $\mathrm{m}^{2}$

$\mathrm{T}_{0} \quad$ the gas reference temperature, $\mathrm{K}$

$\Delta \mathrm{t} \quad$ time step, $\mathrm{s}$

$\mathrm{u}$ the velocity along the axis of the pipe, $\mathrm{m} / \mathrm{s}$ the Cartesian coordinate along the axis of the pipe, $\mathrm{m}$

$$
\begin{array}{ll}
\rho_{0} & \text { the reference density, } \mathrm{kg} / \mathrm{m}^{3} \\
\mu & \text { coefficient of viscosity, } \mathrm{N} \mathrm{s} / \mathrm{m}^{2}
\end{array}
$$

\section{REFERENCE}

Aoki, K., 2001, Dynamics of Rarefied Gas Flows: Asymptotic and Numerical Analyses of the Boltzmann Equation, AIAA 2001-0874.

Bird, G. A., 1994, Molecular Gas Dynamics and the Direct Simulation of Gas Flows, Clarendon Press, Oxford.

Cai, C., Boyd, I. D., Fan, J., and Candler G. V., 2000, Direct Simulation Methods for Low-Speed Microchannel Flows, J. Thermophysics and Heat Transfer, vol. 14, pp. 368-378.

Cercignani, C., and Sernagiotto, F., 1966, Cylindrical Poiseuille Flow of a Rarefied Gas, Phys. Fluids, vol. 9, pp. 40-44.

Fan, J., Boyd, I. D., and Cai, C. P., 2001, Computation of Rarefied Flows around a NACA 0012 Airfoil, AIAA J., vol. 39, pp. 618-625.

Fan, J., and Shen, C., 1999, Statistical Simulation of Low-Speed Unidirectional Flows in Transition Regime, in Rarefied Gas Dynamics, edited by R. Brun et al, Cepadues Editions, Vol. 2, pp. 245-252.

Fan, J., and Shen, C., 2001, Statistical Simulation of Low-Speed Rarefied Gas Flows, J. Comp. Phys., Vol. 167, pp. 393-412.

Hasegawa, M., and Sone, Y., 1988, Poiseuille and Thermal Transpiration Flows of a rarefied gas for various pipes, J. Vac. Soc. Jpn., 31, pp. 416 (in Japanese).

Ho, C.M., and Tai, Y. C., 1998, Micro-ElectroMechanical Systems and Fluid Flows, Annu. Rev. Fluid Mech. 30, 579-612.

Loyalka, S. K., Storvick, T. S., and Park, H. S., 1976, Poiseuille Flow and Thermal Creep Flow in Long, Rectangular Channels in the Molecular and Transition Flow Regimes, J. Vac. Sci. Tech., Vol. 13, 1188-1192.

Ohwada, T., Sone, Y., and Aoki, K., 1989, Numerical Analysis of the Poiseuille and Thermal Transpiration Flows between Two Parallel Plates on the Basis of the Boltzmann Equation for Hard-Sphere Molecules, Phys. Fluids A 1, pp. 2042-2049.

Pong, K. C., Ho, C. M., Liu, J.Q., and Tai, Y. C., 1994, Non-linear Pressure Distribution in Uniform Microchannel, ASME-FED, vol.197, pp51-56.

Sharipov, F., 1999, Rarefied Gas Flow through a Long Rectangular Channel, J. Vac. Sci. Tech., A 17, pp. 3062-3066.

Shih, J.C., Ho, C. M., Liu, J. Q., and Tai, Y. C., 1996, Monoatomic and Polyatomic Gas Flow through Uniform Microchannels, ASME-DSC, vol.59, pp197-203.

Shen, C., 2003, Rarefied Gas Dynamics, National Defence Industry Press, Beijing (in China). 
Shen, C., Fan, J., and Xie, C., 2003, Statistical Simulation of Rarefied Gas Flows in Micro-Channels, to appear in J. Comp. Phys..

Shen, C., Xu, X., Hu, Z., and Wu, W., 1994, Transient motion of rarefied gas caused by heat addition simulated by DSMC method, Progress in Arstro-Aero., Vol. 159, pp.234-242.

Sone, Y., and Yamamoto, 1968, K., Flow of Rarefied Gas through a Circular Pipe, Phys. Fluids, Vol. 11, pp. 1672-1178; Errata: Phys. Fluids, Vol. 13, 1651 (1970).

Sun, Q. H., Boyd, I. D., and Candler, G. V., 2001, Numerical Simulation of Gas Flow over Micro-Scale Airfoils, AIAA 2001-3071.

Vincenti, W. G., Kruger, C. H. Jr, 1965, Introduction to Physical Gas Dynamics, edited by John Wiley \& Sons.

Xie, C., Fan, J., and Shen, C., 2002, Rarefied Gas Flows in Micro-Channels, presented at 23rd rarefied gas symposium. 